\title{
On the Well-Posedness for a Class of Pseudo-Differential Parabolic Equations
}

\author{
Julio Delgado
}

\begin{abstract}
In this work we study the well-posedness of the Cauchy problem for a class of pseudo-differential parabolic equations in the framework of Weyl-Hörmander calculus. We establish regularity estimates, existence and uniqueness in the scale of Sobolev spaces $H(m, g)$ adapted to the corresponding Hörmander classes. Some examples are included for fractional parabolic equations and degenerate parabolic equations.
\end{abstract}

Mathematics Subject Classification. Primary 35L80; Secondary 47G30, 35L40, 35A27.

Keywords. Degenerate parabolic equation, Fractional diffusion,

Nonhomogeneous calculus, Microlocal analysis.

\section{Introduction}

The research on degenerate parabolic equations has been very active in the last decades and developed into multiple directions. A good account on the local structure of solutions and other interests can be found in [11]. On the other hand, fractional diffusion equations have a wide variety of applications e.g. fluid mechanics [6,22], mathematical finance [7] and fractional dynamics $[18,19,26]$.

Here we will establish the well-posedness of the Cauchy problem for a class of pseudo-differential parabolic equations in the setting of WeylHörmander calculus. We will give some examples of applications for fractional degenerate parabolic equations and fractional diffusion equations. The analysis of PDEs under the scrutiny of pseudo-differential calculus has been largely exploited. Some of the main ideas to study parabolic equations in this work correspond to an adaptation of the methods used for the classical classes of pseudo-differential operators as presented for instance in $[5,27,28]$.

We now give a more precise description of the problem studied in this work. Let $g$ be a Hörmander metric $[15,20]$ on the phase space $\mathbb{R}^{n} \times \mathbb{R}^{n}$ and $m$ a $g$-weight, we denote by $S(m, g)$ the associated class of symbols and by $O p S(m, g)$ the corresponding class of pseudodifferential operators.

The author was supported by the Leverhulme Research Grants RPG-2014-02 and RPG2017-151. 
The Kohn-Nirenberg quantization of a symbol $a(x, \xi)$ will be denoted by $a(x, D)$ and the Weyl quantization by $a^{w}$. We will study the Cauchy problem associated to a parabolic equation of the form

$$
\frac{\partial v}{\partial t}=K\left(t, x, D_{x}\right) v
$$

where for every $t \in \mathbb{R}, K\left(t, x, D_{x}\right)$ is a suitable pseudo-differential operator of order 2 relative to a Hörmander metric $g$ (cf. $[14,15,20])$ and a suitable positivity condition is imposed on $\operatorname{Re} K(t, x, \xi)$. We will establish regularity estimates, existence and uniqueness with respect to the Sobolev spaces $H(m, g)$ adapted to the $S(m, g)$ classes. The simplest case corresponds to the heat equation, which is associated to an operator of the form $\frac{\partial}{\partial t}-K$, where $K$ is a suitable elliptic second order differential operator and the considered symbol classes are the ones introduced by Kohn and Nirenberg (cf. [16]). Degenerate hyperbolic pseudo-differential equations have been recently studied in [9] as well as some preliminary results in the parabolic case.

The Planck function or the uncertainty parameter $h(x, \xi)$ associated to a Hörmander metric $g$ is defined by $h(x, \xi)=\sup _{(y, \eta) \neq 0}\left(\frac{g_{(x, \xi)}(y, \eta)}{g_{(x, \xi)}^{\sigma}(y, \eta)}\right)^{1 / 2}$, where $g^{\sigma}$ is the dual metric of $g$ with respect to the canonical symplectic form $[(y, \eta),(z, \zeta)]=z \cdot \eta-y \cdot \zeta$. This function can be used to extend the notion of operators of order $\nu$ for a real number $\nu$ (cf. Sect. 2). Indeed, the weight $h(x, \xi)^{-\nu}$ will correspond to the order $\nu$.

The main theorem of this work can be described as follows. Given a Hörmander metric $g$, we study the following Cauchy problem for $K(t, x, \xi) \in$ $S\left(h^{-\nu}, g\right)$ with $\nu \in\{1,2\}$ and write $K(t)=K\left(t, x, D_{x}\right)$ :

$$
\left\{\begin{aligned}
\frac{\partial v}{\partial t} & =K(t) v+\omega, \quad\left(\text { in the sense of } \mathcal{D}^{\prime}(] 0, T\left[\times \mathbb{R}^{n}\right)\right) \\
v(0) & =f
\end{aligned}\right.
$$

where $f \in H\left(h^{-s}, g\right), \omega \in L^{2}\left([0, T], H\left(h^{-(s-\nu+1)}, g\right)\right)$ for some $s \in \mathbb{R}$ and a suitable positivity condition is imposed on $K$. Under such assumptions we will prove the existence and uniqueness of a solution $v \in C\left([0, T], H\left(h^{-s}, g\right)\right)$.

An important example of an operator of order 2 with respect to $g$ is given by $\Delta_{(g)} \in O p S\left(h^{-2}, g\right)$ of the form

$$
\Delta_{(g)}:=h^{-2}(x, D)+R(x, D),
$$

where $R \in O p S\left(h^{-1}, g\right)$. A basic example is the one of the metric $g=g^{1,0}=$ $d x^{2}+\frac{d \xi^{2}}{\langle\xi\rangle^{2}}$, where $\langle\xi\rangle^{2}=1+|\xi|^{2}$. In that case one has $h^{-2}(x, D)=-\Delta+I$, where $\Delta$ is the Laplacian on $\mathbb{R}^{n}$ and $I$ the identity operator. By choosing $K(t)=-\Delta_{(g)}=\Delta-I$ and $R(x, D)=I$ in (1.2), (1.1) becomes the classical Cauchy problem for the heat equation. In general, the operator $\Delta_{(g)}$ is not elliptic and there is where the difficulty arises. Indeed this operator does not even have a parametrix as a classical pseudodifferential operator. In this situation the Weyl-Hörmander calculus is appropriate to deal with it. The type of estimates here obtained for the problem (1.1) are well known when 
the operator $\Delta_{(g)}$ is replaced by a second order partial differential operator $P\left(t, x, D_{x}\right)$ such that $\frac{\partial}{\partial t}+P$ is strictly parabolic.

By choosing a suitable Hörmander metric one can consider for instance $-K$ of the form: (i) $K(D)=-(-\Delta+I)^{\ell}$ for $0<\ell \leq 1$. A further analysis of this example will allow us to study fractional diffusion equations. Strictly speaking the fractional Laplacian $(-\Delta)^{s} 0<s<1$ has not a symbol in a class of symbols for pseudo-differential operators. However we can decompose it in the form $(-\Delta)^{s}=\sigma(x, D)+E$ so that the main term $\sigma(x, D)$ is a pseudodifferential operator and $E$ has some good properties allowing to extend our analysis to the fractional Laplacian. The underlying ideas for such splitting has been recently applied in [12].

(ii) $K(x, D)=-\left((-\Delta)^{2}+|x|^{4}\right)$; higher order parabolic equations have been investigated for the last fifty years and it is well known that they exhibit different pathologies with respect to the classical case. For instance the kernels of the heat operators depends on the space dimension and they are not expressible in a simple form. Another pathology is the lack of the positivity preserving property (cf. $[1,13,21]$ and the references therein).

(iii) $K(x, D)=\partial_{x_{1}}^{2}+\widetilde{x}_{1}^{2} \partial_{x_{2}}^{2}-C$ on $\mathbb{R}^{2}$, where $C>0$ is large enough; $\widetilde{x}$ is in the class $\mathbb{C}^{\infty}(\mathbb{R}), \widetilde{x}=x$ for $|x| \leq 2, \widetilde{x}$ is increasing, $\widetilde{x}=\omega \operatorname{sgn}(x)$ if $|x| \geq 4$, for a constant $\omega \geq 4$; (iv) $K(x, D)=-\left(L^{2}+J^{2 \tau}\right) J^{-2}$, where $L$ is a second order differential operator with index of sub-ellipticity $\tau$ as in (3.6) and $J$ is the Bessel potential of order 1 on $\mathbb{R}^{n}$, i.e. corresponding to the symbol $\langle\xi\rangle$. The operators in (iii) are subelliptic with order of subellipticity $\tau=1$ since the associated vector fields to the sum of squares satisfy the Hörmander condition of order 2. They have been studied in [29] C-J. Xu and $\mathrm{X}$. Zhu, where the authors prove that $K(x, D)-C$ is invertible in a suitable nonhomogeneous class for $C>0$ large enough. The operator $K(x, D)$ can be seen as a particular case of $Q=\sum_{i=1}^{n} \partial_{x_{i}}^{2}+|x|^{2 k} \sum_{i=1}^{m} \partial_{t_{i}}^{2}-C$ on $\mathbb{R}^{n+m}$ for which, Beals, Gaveau and Greiner have found exact fundamental solutions (cf. [2]).

We also point out that parabolic equations (possibly completely degenerate) in the case of a constant Weyl-Hormander metric (hence corresponding to the symbol classes $S_{0,0}^{0}$ ) were also addressed in [23], where a representation of the propagator as pseudodifferential operator with symbol in the same class was proved as well. We hope to consider such a representation in a second paper.

\section{Preliminaries}

In this section we review some elements of the Weyl-Hörmander calculus. For a comprehensive study on this important theory we refer the reader to $[4,15,20]$.

The Kohn-Nirenberg and Weyl quantizations are recalled below:

Definition 2.1. For $a(x, \xi) \in S^{\prime}\left(\mathbb{R}^{n} \times \mathbb{R}^{n}\right)\left(x \in \mathbb{R}^{n}\right.$ and $\left.\xi \in \mathbb{R}^{n}\right)$, we define the Kohn-Nirenberg or classic quantization as the operator $a(x, D): S\left(\mathbb{R}^{n}\right) \rightarrow$ $S\left(\mathbb{R}^{n}\right)$ given by 


$$
a(x, D) u(x)=(2 \pi)^{-n} \iint e^{i(x-y) \xi} a(x, \xi) u(y) d y d \xi .
$$

The Weyl quantization has a fundamental relationship with the symplectic structure of $\mathbb{R}^{n} \times \mathbb{R}^{n}=T^{*} \mathbb{R}^{n}$ as we will see below. The Weyl quantisation of $a(x, \xi)$, is given by the operator $a^{w}: S\left(\mathbb{R}^{n}\right) \rightarrow S\left(\mathbb{R}^{n}\right)$ where

$$
a^{w} u(x)=(2 \pi)^{-n} \iint e^{i(x-y) \xi} a\left(\frac{x+y}{2}, \xi\right) u(y) d y d \xi .
$$

Definition 2.2. Let $a(x, \xi), b(x, \xi) \in S\left(\mathbb{R}^{n} \times \mathbb{R}^{n}\right)$ we define

$$
(a \# b)(X)=\pi^{-2 n} \int_{\mathbb{R}^{2 n} \times \mathbb{R}^{2 n}} e^{-2 i \sigma\left(X-Y_{1}, X-Y_{2}\right)} a\left(Y_{1}\right) b\left(Y_{2}\right) d Y_{1} d Y_{2},
$$

where $\sigma(X, Y)=y \cdot \xi-x \cdot \eta$ for $X=(x, \xi)$ and $Y=(y, \eta)$.

The operation \# is useful in order to describe the composition $a^{w} \circ$ $b^{\omega}$, indeed one has $a^{w} \circ b^{\omega}=(a \# b)^{\omega}$. Moreover, from the formula for the composition we notice how the symplectic form naturally appears in the integral expression above.

We shall now recall the definition of Hörmander metrics on the phasespace. A Hörmander metric is a special Riemannian metric which carries basic information of the differential operator target into the phase space.

Definition 2.3. For $X \in \mathbb{R}^{n} \times \mathbb{R}^{n}$ let $g_{X}(\cdot)$ be a positive definite quadratic form on $\mathbb{R}^{n} \times \mathbb{R}^{n}$, we say that $g(\cdot)$ is a Hörmander's metric if the following three conditions are satisfied:

1. Continuity - There exist constants $C, c, c^{\prime} \in \mathbb{R}$ such that $g_{X}(Y) \leq C$, for $X, Y \in \mathbb{R}^{n} \times \mathbb{R}^{n}$, implies $c^{\prime} \cdot g_{X+Y}(T) \leq g_{X}(T) \leq c \cdot g_{X+Y}(T)$ for every $T \in \mathbb{R}^{n} \times \mathbb{R}^{n}$.

2. Uncertainty principle-For $Y=(y, \eta)$ and $Z=(z, \zeta)$ we define $\sigma(Y, Z)=$ $z \cdot \eta-y \cdot \zeta$, and

$$
g_{X}^{\sigma}(T)=\sup _{W \neq 0} \frac{\sigma(T, W)^{2}}{g_{X}(W)} .
$$

We say that $g$ satisfies the uncertainty principle if

$$
\lambda_{g}(X)=\inf _{T \neq 0}\left(\frac{g_{X}^{\sigma}(T)}{g_{X}(T)}\right)^{1 / 2} \geq 1
$$

for all $X \in \mathbb{R}^{n} \times \mathbb{R}^{n}$.

3. Temperateness - We say that $g$ is temperate if there exist $\bar{C}>0$ and $J \in \mathbb{N}$ such that

$$
\left(\frac{g_{X}(\cdot)}{g_{Y}(\cdot)}\right)^{ \pm 1} \leq \bar{C}\left(1+g_{Y}^{\sigma}(X-Y)\right)^{J}
$$

Let $g$ be a Hörmander's metric, the uncertainty parameter or the Planck function associated to $g$ is defined by

$$
h(X)^{2}=\sup _{T \neq 0} \frac{g_{X}(T)}{g_{X}^{\sigma}(T)}
$$

and it is clear that $h(X)=\left(\lambda_{g}(X)\right)^{-1}$. 
The uncertainty principle can be translated then by the condition

$$
h(X) \leq 1
$$

Remark 2.4. (i) For a split metric g, i.e. of type

$$
g_{X}(d x, d \xi)=\sum_{i=1}^{n} \frac{d x_{i}^{2}}{a_{i}(X)}+\frac{d \xi_{i}^{2}}{b_{i}(X)},
$$

where $a_{i}(X)$ and $b_{i}(X)$ are positive functions, one can prove

$$
g_{X}^{\sigma}(d x, d \xi)=\sum_{i=1}^{n} b_{i}(X) d x_{i}^{2}+a_{i}(X) d \xi_{i}^{2} .
$$

(ii) A special case of (i) is the one of symmetrically split metric i.e. of type

$$
g_{X}(d x, d \xi)=\frac{d x^{2}}{a(X)}+\frac{d \xi^{2}}{b(X)} .
$$

The metric (3.7) is an example of a such type.

(iii) If $g$ is a split metric one can prove the following formula for $\lambda_{g}$

$$
\lambda_{g}(X)=\min _{j} \sqrt{a_{j}(X) b_{j}(X)} .
$$

(iv) In particular, if $g$ is symmetrically split like in (ii) then

$$
\lambda_{g}(X)=\sqrt{a(X) b(X)} .
$$

The classical weight $\langle\xi\rangle^{m}$ is generalised in the following way for a corresponding Hörmander metric.

Definition 2.5. We say that a strictly positive function $M$ is a $g$-weight or $g$-continuous if there exists $\tilde{C}>0$ and $N \in \mathbb{N}$ such that

$$
\left(\frac{M(X+Y)}{M(X)}\right)^{ \pm 1} \leq \tilde{C}, \quad \text { if } g_{X}(Y) \leq \frac{1}{\tilde{C}}
$$

We say that $M$ is $g$-temperate if there exists $C>0$ and $N \in \mathbb{N}$ such that

$$
\left(\frac{M(Y)}{M(X)}\right)^{ \pm 1} \leq \tilde{C}\left(1+g_{Y}^{\sigma}(X-Y)\right)^{N} .
$$

Definition 2.6. For a Hörmander metric $g$ and a $g$-weight $M$, we denote by $S(M, g)$ the set of all smooth functions $a$ on $\mathbb{R}^{n} \times \mathbb{R}^{n}$ such that for any integer $k$ there exists $C_{k} \in R$, such that for all $X, T_{1}, \ldots, T_{k} \in \mathbb{R}^{n} \times \mathbb{R}^{n}$

$$
\left|a^{(k)}\left(X ; T_{1}, \ldots, T_{k}\right)\right| \leq C_{k} M(X) \prod_{i=1}^{k} g_{X}^{1 / 2}\left(T_{i}\right) .
$$

For $a \in S(M, g)$ we denote by $\|a\|_{k, S(M, g)}$ the minimum $C_{k}$ satisfying the above inequality. The class $S(M, g)$ becomes a Frechet space endowed with the family of seminorms $\|\cdot\|_{k, S(M, g)}$. 
Remark 2.7. Given a Hörmander metric $g$. Instead of use the Planck function for the formulation of the statements we can equivalently employ the weight $\lambda_{g}$ defined by $\lambda_{g}=h^{-1}$. The function $\lambda_{g}$ is a $g$-weight for the metric $g$ (cf. [15]). Given a $g$-weight $M$, it is possible to construct an equivalent smooth weight $\widetilde{M}$ such that $\widetilde{M} \in S(M, g)$ (cf. [15,20]). In particular, for $\lambda_{g}$ there exists an equivalent smooth weight $\widetilde{\lambda_{g}}$ such that $\widetilde{\lambda_{g}} \in S\left(\lambda_{g}, g\right)$. Hence, $\widetilde{\lambda_{g}} \in$ $S\left(\widetilde{\lambda_{g}}, g\right)$. A weight $M$ such that $M \in S(M, g)$ is called regular. Thus the weight $\lambda_{g}$ and consequently the Planck function $h$ can be assumed to be regulars.

If $g=g^{\rho, \delta}$ is a $(\rho, \delta)$-metric, we have

$$
\lambda_{g}(X)=\langle\xi\rangle^{(\rho-\delta)} .
$$

In the special case $\rho=1, \delta=0$ then one has $\lambda_{g}(X)=\langle\xi\rangle$. The weight $\lambda_{g}$ can be seen as an extension of the basic one $\langle\xi\rangle$, for the $(\rho, \delta)$ classes. The symbols in $S\left(\lambda_{g}^{\mu}, g\right)$ for $\mu \in \mathbb{R}$ can be seen as the symbols of order $\mu$ with respect to the metric $g$. In particular, $\lambda_{g}^{\mu}$ is a symbol of order $\mu$ with respect to $g$.

We recall the following theorem which gives the asymptotic expansion for the composition of two symbols. The proof can also be found in [4] or [20].

Theorem 2.8. Let $g$ be a Hörmander metric on $\mathbb{R}^{n} \times \mathbb{R}^{n}, M_{1}, M_{2}$ two $g$ weights. If $a \in S\left(M_{1}, g\right), b \in S\left(M_{2}, g\right)$, then for all $N \in \mathbb{N}$,

$$
a \# b-\left.\sum_{0 \leq j<N} \frac{1}{j !}\left(i\left[D_{X_{1}}, D_{X_{2}}\right]\right)^{j}(a \otimes b)\right|_{\text {diagonal }} \in S\left(M_{1} M_{2} \lambda_{g}^{-N}, g\right) \text {. }
$$

We now recall the definition of Sobolev spaces adapted to the WeylHörmander calculus. Here we adopt the Beals's definition for simplicity in the presentation of the basic theory. Comprehensive treatments on Sobolev spaces in this setting can be found in $[3,20]$.

Definition 2.9. Let $g$ be a Hörmander metric and $M$ a $g$-weight. We will call Sobolev space relative to $M$ and it will be denoted by $H(M, g)$, the set of tempered distributions $u$ on $\mathbb{R}^{n}$ such that

$$
a^{w} u \in L^{2}, \forall a \in S(M, g) .
$$

Remark 2.10. We observe that the definition above requires a test over all the symbols in $S(M, g)$. In contrast, we note that the classical Sobolev spaces $H^{m}=H\left(\langle\xi\rangle^{m}, g^{1,0}\right)$ are defined by the condition on the tempered distribution $u$ :

$$
a(x, D) u \in L^{2}, \text { with } a(x, \xi)=\langle\xi\rangle^{m} .
$$

This means that, in the classical case the Sobolev space is defined by a fixed symbol. This property can be generalised to the Sobolev spaces $H(M, g)$. Indeed, that is a consequence of the existence (cf. [3]), for every $g$-weight $M$ of a one parameter group (for the operation \#) of symbols $b_{t} \in S\left(M^{t}, g\right)$. Thus, $b_{s} \# b_{t}=b_{s+t}$ for all $s, t \in \mathbb{R}$. In particular: there exist $b \in S(M, g)$ and 
$b^{\prime} \in S\left(M^{-1}, g\right)$, such that $b \# b^{\prime}=b^{\prime} \# b=1$. Moreover, for every $g$-weight $M_{1}$, the operator $b^{w}$ is an isomorphism from $H\left(M_{1}, g\right)$ onto $H\left(M_{1} / M, g\right)$. For a such $b$ and a tempered distribution $u \in \mathcal{S}^{\prime}\left(\mathbb{R}^{n}\right)$ one has

$$
u \in H(M, g) \text { if and only if } b^{w} u \in L^{2}\left(\mathbb{R}^{n}\right) .
$$

The characterisation (2.5) is very useful for the analysis carried out to obtain the main results.

The action of the Weyl quantization on the Sobolev spaces is determined by the following theorem (cf.[3]).

Theorem 2.11. Let $g$ be a Hörmander metric, $M$ and $M_{1}$ be two g-weights. For every $a \in S(M, g)$, we have

$$
a^{w}: H\left(M_{1}, g\right) \rightarrow H\left(M_{1} / M, g\right) .
$$

It is customary to identify $H(1, g)(M=1)$ with $L^{2}$ (cf.[3]):

Theorem 2.12. For a Hörmander's metric $g$ we have $H(1, g)=L^{2}$.

\section{Results}

In this section we prove the results of this work. First, we consider energy estimates for parabolic equations of order 1 and 2 relative to a Hörmander metric $g$. Second, we establish the existence, regularity and uniqueness with respect to $H(m, g)$ spaces for the problem (1.1) and give some examples. We consider the cases $\nu=1$ and $\nu=2$ separately since the corresponding energy inequalities that we will obtain are of different nature.

In the following theorems we will write $K(t, x, \xi) \in S\left(\lambda_{g}^{\nu}, g\right)(\nu=1,2)$, which should be understood in the sense that for each $t \in \mathbb{R}$ fixed, $K(t, \cdot, \cdot) \in$ $S\left(\lambda_{g}^{\nu}, g\right)$. For $t$ fixed, $K\left(t, x, D_{x}\right)=K(t)$ will denote the pseudo-differential operator corresponding to $K(t, x, \xi)$.

\subsection{Case of order $\nu=1$}

We start with an energy estimate for parabolic equations of order 1 in the $S(M, g)$-calculus. Gårding inequalities in the setting of $S(M, g)$ classes will play an essential role.

Theorem 3.1. Let $g$ be a Hörmander metric, $T>0, K(t, x, \xi) \in S\left(\lambda_{g}^{1}, g\right)$ depending smoothly on $t$ and $\operatorname{Re}(-K) \geq 0$. If $v \in C\left([0, T], H\left(\lambda_{g}^{s+1}, g\right)\right) \cap$ $C^{1}\left([0, T], H\left(\lambda_{g}^{s}, g\right)\right)$ for some $s \in \mathbb{R}$ and $Q=\partial_{t}-K(t)$. Then $v$ satisfies

$$
\|v(t)\|_{H\left(\lambda_{g}^{s}, g\right)}^{2} \leq e^{C t}\left(\|v(0)\|_{H\left(\lambda_{g}^{s}, g\right)}^{2}+\int_{0}^{t}\|Q v(\tau)\|_{H\left(\lambda_{g}^{s}, g\right)}^{2} d \tau\right)
$$

for all $t \in[0, T]$. Moreover, we can replace $v(0)$ by $v(T)$ on the right-hand side of (3.1). The same conclusion holds for the operator $Q^{*}$. 
Proof. We now assume that $v \in C\left([0, T], H\left(\lambda_{g}^{s+1}, g\right)\right) \cap C^{1}\left([0, T], H\left(\lambda_{g}^{s}, g\right)\right)$ and write $\omega=Q v$. We will consider the characterisation of Sobolev spaces $H\left(\lambda_{g}^{s}, g\right)$ via the one parameter group $b_{s}$ as described by Remark 2.10. We will write the Kohn-Nirenberg quantization of $b_{s}$ as $b_{s}(x, D)=\Lambda_{g}^{s}$. By (2.5) with $M=\lambda_{g}$, a tempered distribution $u$ belongs to $H\left(\lambda_{g}^{s}, g\right)$ if and only if $\Lambda_{g}^{s} u \in L^{2}\left(\mathbb{R}^{n}\right)$. Since $\partial_{t} v=\left(\partial_{t}-K(t)\right) v+K(t) v=Q v+K(t) v=\omega+K(t) v$, we observe that

$$
\begin{aligned}
\frac{d}{d t}\|v(t)\|_{H\left(\lambda_{g}^{s}, g\right)}^{2}= & \frac{d}{d t}\left\langle\Lambda_{g}^{s} v, \Lambda_{g}^{s} v\right\rangle \\
= & 2 \operatorname{Re}\left\langle\Lambda_{g}^{s} v_{t}, \Lambda_{g}^{s} v\right\rangle \\
= & 2 \operatorname{Re}\left\langle\Lambda_{g}^{s}(K(t) v+\omega), \Lambda_{g}^{s} v\right\rangle \\
= & 2 \operatorname{Re}\left\langle\Lambda_{g}^{s} K(t) v, \Lambda_{g}^{s} v\right\rangle \\
& -2 \operatorname{Re}\left\langle K(t) \Lambda_{g}^{s} v, \Lambda_{g}^{s} v\right\rangle \\
& +2 \operatorname{Re}\left\langle K(t) \Lambda_{g}^{s} v, \Lambda_{g}^{s} v\right\rangle \\
& +2 \operatorname{Re}\left\langle\Lambda_{g}^{s} \omega, \Lambda_{g}^{s} v\right\rangle \\
= & 2 \operatorname{Re}\left\langle\left[\Lambda_{g}^{s}, K(t)\right] v, \Lambda_{g}^{s} v\right\rangle \\
& +2 \operatorname{Re}\left\langle K(t) \Lambda_{g}^{s} v, \Lambda_{g}^{s} v\right\rangle \\
& +2 \operatorname{Re}\left\langle\Lambda_{g}^{s} \omega, \Lambda_{g}^{s} v\right\rangle .
\end{aligned}
$$

The term (3.2) can be written in the following way

$$
\begin{aligned}
2 \operatorname{Re}\left\langle K(t) \Lambda_{g}^{s} v, \Lambda_{g}^{s} v\right\rangle & =\left\langle K(t) \Lambda_{g}^{s} v, \Lambda_{g}^{s} v\right\rangle+\overline{\left\langle K(t) \Lambda_{g}^{s} v, \Lambda_{g}^{s} v\right\rangle} \\
& =\left\langle K(t) \Lambda_{g}^{s} v, \Lambda_{g}^{s} v\right\rangle+\left\langle\Lambda_{g}^{s} v, K(t) \Lambda_{g}^{s} v\right\rangle \\
& =\left\langle K(t) \Lambda_{g}^{s} v, \Lambda_{g}^{s} v\right\rangle+\left\langle K(t)^{*} \Lambda_{g}^{s} v, \Lambda_{g}^{s} v\right\rangle \\
& =\left\langle\left(K(t)+K(t)^{*}\right) \Lambda_{g}^{s} v, \Lambda_{g}^{s} v\right\rangle .
\end{aligned}
$$

Now, we have $A(t)=\left[\Lambda_{g}^{s}, K(t)\right] \in O p S\left(\lambda_{g}^{s}, g\right)$. Since $0 \leq \operatorname{Re}(-K(t, x, \xi)) \in$ $S\left(\lambda_{g}^{1}, g\right)$ by the sharp Garrding inequality (cf. Theorem 18.6.7. [15]), it follows that

$$
\begin{aligned}
& \frac{d}{d t}\|v(t)\|_{H\left(\lambda_{g}^{s}, g\right)}^{2} \\
& \quad \leq\|A(t) v\|_{L^{2}}\|v\|_{H\left(\lambda_{g}^{s}, g\right)}+C_{1}\|v\|_{H\left(\lambda_{g}^{s}, g\right)}^{2}+C_{2}\|\omega\|_{H\left(\lambda_{g}^{s}, g\right)}\|v\|_{H\left(\lambda_{g}^{s}, g\right)} \\
& \quad \leq C\|v\|_{H\left(\lambda_{g}^{s}, g\right)}\|v\|_{H\left(\lambda_{g}^{s}, g\right)}+C_{1}\|v\|_{H\left(\lambda_{g}^{s}, g\right)}^{2}+C_{2}\|\omega\|_{H\left(\lambda_{g}^{s}, g\right)}\|v\|_{H\left(\lambda_{g}^{s}, g\right)} \\
& \quad \leq C\|v\|_{H\left(\lambda_{g}^{s}, g\right)}^{2}+C\|\omega\|_{H\left(\lambda_{g}^{s}, g\right)}^{2} .
\end{aligned}
$$

An application of the Gronwall inequality gives us the following energy inequality

$$
\|v(t)\|_{H\left(\lambda_{g}^{s}, g\right)}^{2} \leq e^{C t}\left(\|v(0)\|_{H\left(\lambda_{g}^{s}, g\right)}^{2}+\int_{0}^{t}\|\omega(\tau)\|_{H\left(\lambda_{g}^{s}, g\right)}^{2} d \tau\right) .
$$

We can also prove an analogous estimate with $v(T)$ instead of $v(0)$ on the right-hand side of the inequality (3.3). Since $\operatorname{Re}\left(K^{*}\right)=\operatorname{Re}(K)$ one can note that the same conclusion holds for $Q^{*}$. 
As an application of the above estimates we will establish a theorem on the existence, uniqueness and regularity in the case of order 1 . We will also required of the following spaces $H_{g}^{+\infty}=\bigcap_{s \in \mathbb{R}} H\left(\lambda_{g}^{s}, g\right)$ and $H_{g}^{-\infty}=\bigcup_{s \in \mathbb{R}} H\left(\lambda_{g}^{s}, g\right)$.

Theorem 3.2. Let $g$ be a Hörmander metric. Let $K(t, x, \xi) \in S\left(\lambda_{g}, g\right)$ depending smoothly on $t$ and $\operatorname{Re}(-K) \geq 0$. Let $s \in \mathbb{R}, T>0, f \in H\left(\lambda_{g}^{s}, g\right), \omega \in$ $L^{2}\left([0, T], H\left(\lambda_{g}^{s}, g\right)\right)$. Then, there exists a unique $v \in C\left([0, T], H\left(\lambda_{g}^{s}, g\right)\right)$ such that

$$
\left\{\begin{aligned}
\frac{\partial v}{\partial t} & =K(t) v+\omega, \quad\left(\text { in the sense of } \mathcal{D}^{\prime}(] 0, T\left[\times \mathbb{R}^{n}\right)\right) \\
v(0) & =f
\end{aligned}\right.
$$

Moreover, the solution $v$ satisfies the energy estimate (3.1). If $\omega \in$ $C^{\infty}\left([0, T], H_{g}^{+\infty}\right)$ and $f \in H_{g}^{+\infty}$ then $v \in C^{\infty}\left([0, T], H_{g}^{+\infty}\right)$.

Proof. We now prove the existence of a solution $v$ of $(3.4)$ in $C([0, T]$, $\left.H\left(\lambda_{g}^{s}, g\right)\right)$. The main arguments here are adapted from the corresponding part in the proof of Theorem 4.5, Chap. 6 in [5]. We write $Q=\frac{\partial}{\partial t}-K$ and we introduce the space $E=\left\{\varphi \in C^{\infty}\left([0, T], H_{g}^{-\infty}\right) \mid \varphi(T)=0\right\}$. We will see that we can define a linear form $\beta$ on $Q^{*} E$ by

$$
Q^{*} \varphi \rightarrow \beta\left(Q^{*} \varphi\right)=\int_{0}^{T}(\omega(t, \cdot), \varphi(t, \cdot)) d t+\frac{1}{i}(f, \varphi(0, \cdot)) .
$$

We note that the energy estimate (3.1) holds for $-s$ for the Cauchy problem (3.4) corresponding to the operator $Q^{*}$ with $\|v(T, \cdot)\|_{H\left(\lambda_{g}^{s}, g\right)}$ on the right hand side of (3.1). Thus, for $\varphi \in E$ we have

$$
\|\varphi(t, \cdot)\|_{H\left(\lambda_{g}^{-s}, g\right)}^{2} \leq C \int_{0}^{T}\left\|Q^{*} \varphi\left(t^{\prime}, \cdot\right)\right\|_{H\left(\lambda_{g}^{-s}, g\right)}^{2} d t^{\prime}, \quad t \in[0, T],
$$

so that

$$
\left|\beta\left(Q^{*} \varphi\right)\right|^{2} \leq C^{\prime} \int_{0}^{T}\left\|Q^{*} \varphi\left(t^{\prime}, \cdot\right)\right\|_{H\left(\lambda_{g}^{-s}, g\right)}^{2} d t^{\prime} .
$$

We deduce that $\beta$ is well defined and continuous with respect to the topology induced on $Q^{*} E$ by $L^{2}\left([0, T],, H\left(\lambda_{g}^{-s}, g\right)\right)$. An application of the HahnBanach theorem implies the existence of an element $v \in\left(L^{2}([0, T\right.$,$] ,$ $\left.\left.H\left(\lambda_{g}^{-s}, g\right)\right)\right)^{\prime}=L^{2}\left([0, T],, H\left(\lambda_{g}^{s}, g\right)\right)$ such that

$$
\left(v, Q^{*} \varphi\right)=\int_{0}^{T}(\omega(t, \cdot), \varphi(t, \cdot)) d t+\frac{1}{i}(f, \varphi(0, \cdot))
$$

for all $\varphi \in E$. In particular, if $\varphi \in C_{0}^{\infty}(] 0, T\left[\times \mathbb{R}^{n}\right)$, (3.5) implies that $Q v=$ $\omega$ in $\left.\mathcal{D}^{\prime}\left([0, T] \times \mathbb{R}^{n}\right)\right)$. Thus $\frac{\partial}{\partial t} v=K v+\omega \in L^{2}\left([0, T],, H\left(\lambda_{g}^{s-1}, g\right)\right)$. An integration by parts with respect to $t$ in $(3.5)$ implies that $(v(0, \cdot), \varphi(0, \cdot))=$ $(f, \varphi(0, \cdot))$ for all $\varphi \in E$ and consequently $v(0)=v(0, \cdot)=f$. 
Now, if $\omega \in C^{\infty}\left([0, T], H_{g}^{+\infty}\right)$ and $f \in H_{g}^{+\infty}$, the above argument shows that $v \in C\left([0, T], H_{g}^{+\infty}\right)$. Moreover, since $\frac{\partial}{\partial t} v=K v+\omega$, one can deduce step by step that $v \in C^{k}\left([0, T], H_{g}^{+\infty}\right)$ for all $k \geq 0$. Consequently $v \in$ $C^{\infty}\left([0, T], H_{g}^{+\infty}\right)$.

We will now prove that $v \in C\left([0, T], H\left(\lambda_{g}^{s}, g\right)\right)$ and that it satisfies the energy estimate (3.1). Suppose we have sequences $(\omega)_{j}$ in $C_{0}^{\infty}\left([0, T] \times \mathbb{R}^{n}\right)$ and $(f)_{j}$ in $C_{0}^{\infty}\left(\mathbb{R}^{n}\right)$ such that $\omega_{j} \rightarrow \omega$ in $L^{2}\left([0, T],, H\left(\lambda_{g}^{s}, g\right)\right)$ and $f_{j} \rightarrow f$ in $H\left(\lambda_{g}^{s}, g\right)$. Let $v_{j} \in C^{\infty}\left([0, T], H_{g}^{+\infty}\right)$ be the solution of $Q v_{j}=\omega_{j}, v_{j}(0, \cdot)=f_{j}$. The inequality (3.1) applied to the $v_{j}-v_{k}$ shows that $v_{j}$ is a Cauchy sequence in $C\left([0, T], H\left(\lambda_{g}^{s}, g\right)\right)$ so that $v_{j} \rightarrow \widetilde{v}$ in $C\left([0, T], H\left(\lambda_{g}^{s}, g\right)\right)$. In the limit, we have $Q \widetilde{v}=\omega, \widetilde{v}(0, \cdot)=f$; consequently, the uniqueness shows that $\widetilde{v}=v$.

The corresponding inequality (3.1) for $v$ is obtained passing to the limit in this inequality applied to $v_{j}$. In this way we conclude the proof of the Theorem. The uniqueness of the solution $v$ follows from the energy inequality (3.1).

Now, coming back to the problem (1.1), we first note that for a given Hörmander metric $g$ we can choose $\widetilde{\lambda_{g}}$ equivalent to $\lambda_{g}$ and regular. In particular, $\widetilde{\lambda_{g}}$ is a smooth symbol and we can justify the use of quantization for the Planck function in (1.2) by writing

$$
\Delta_{(g)}:={\widetilde{\lambda_{g}}}^{2}(x, D)+R(x, D),
$$

where $R \in O p S\left(\lambda_{g}, g\right)$.

We note that if two $g$-weights $M_{1}$ and $M_{2}$ are equivalent, then $S\left(M_{1}, g\right)$ $=S\left(M_{2}, g\right)$ and $H\left(M_{1}, g\right)=H\left(M_{2}, g\right)$. In particular, we have $S\left(\lambda_{g}^{s}, g\right)=$ $S\left(\widetilde{{\lambda_{g}}^{s}}, g\right)$ and $H\left(\lambda_{g}^{s}, g\right)=H\left(\widetilde{\lambda_{g}}, g\right)$ for all $s \in \mathbb{R}$.

Example 1. We give some examples of operators of order $\mu \in \mathbb{R}$ for different metrics and corresponding examples satisfying the conditions of Theorem 3.2:

(i) Let $L$ be a second order differential operator, formally self-adjoint,

$$
L f=-\sum a_{i j}(x) \frac{\partial^{2}}{\partial x_{i} \partial x_{j}} f+\text { lower order terms, } f \in C_{0}^{\infty}\left(\mathbb{R}^{n}\right),
$$

where $\left(a_{i j}(x)\right)$ is a positive semi-definite matrix of real functions and $a_{i j} \in C_{b}^{\infty}\left(\mathbb{R}^{n}\right)\left(C^{\infty}\right.$ functions which are bounded as well as all their derivatives). If $L$ is subelliptic with order of subellipticity $1 \leq \tau \leq 2$, we associate to $L$ the Hörmander metric $g$ defined by

$$
g_{X}(d x, d \xi)=m(X)^{-2}\left(\langle\xi\rangle^{2} d x^{2}+d \xi^{2}\right), \quad\left(X=(x, \xi) \in \mathbb{R}^{2 n}\right)
$$

where

$$
m(X)=m(x, \xi)=\left(a(x, \xi)+\langle\xi\rangle^{\tau}\right)^{\frac{1}{2}},
$$

$\langle\xi\rangle=\left(1+|\xi|^{2}\right)^{\frac{1}{2}}$ and $a(x, \xi)$ is the principal symbol of $L$. The invertibility of $L+C$ in the setting of $S(m, g)$ classes has been studied in [8]. The case $0<\tau \leq 1$ has been investigated for special highly degenerate Grushin operators in [29] and [10]. The study of invertibility requires of lower bounds estimates in a crucial way $[24,25]$. Fundamental solutions 
for highly degenerate elliptic operators (or in other terms small $\tau$ ) have been obtained in [2].

For $L$ as in (3.6) and the metric (3.7), the parameter $\lambda_{g}$ is given by $\lambda_{g}(x, \xi)=\frac{a(x, \xi)+\langle\xi\rangle^{\tau}}{\langle\xi\rangle}$. An example of an operator operator of order $\mu \in \mathbb{R}$ is given by $L_{(g)}^{\mu}$ of the form

$$
L_{(g)}=\left(L+J^{\tau}\right)^{\mu} J^{-\mu}+R(x, D),
$$

where $R(x, D) \in O p S\left(\left(\frac{a(x, \xi)+\langle\xi\rangle^{\tau}}{\langle\xi\rangle}\right)^{\mu-1}, g\right)$ and $J$ is the Bessel potential of order 1 on $\mathbb{R}^{n}$, i.e. corresponding to the symbol $\langle\xi\rangle$ and $R(x, D) \in O p S\left(\lambda_{g}, g\right)$.

(ii) Let $0<\ell \leq 1$ and $g$ be the metric defined by

$$
g=\frac{d x^{2}}{\left(1+|x|^{2}+|\xi|^{2}\right)^{\ell}}+\frac{d \xi^{2}}{\left(1+|x|^{2}+|\xi|^{2}\right)^{\ell}} .
$$

In the case $\ell=1$ it is well known that $g$ is a Hörmander metric [20]. In the general case, the uncertainty principle follows immediately. The slowness follows from the fact that $0<\ell \leq 1$ and the case $\ell=1$. The temperateness is a consequence of the slowness for this type of metric (cf. Example 2, P. 378 of [14] and [20]). In this case $\lambda_{g}=\left(1+|x|^{2}+|\xi|^{2}\right)^{\ell}$ and one can apply Theorem 3.2 to the operator $K\left(x, D_{x}\right)=-(-\Delta+$ $\left.|x|^{2}+1\right)^{\ell}$. The point of view of order with respect to a Hörmander metric $g$ allows us to consider some operators $K\left(t, x, D_{x}\right)$ that being of order 1 with respect to $g$ are also of order $\ell$ as differential operators. For instance that will be the case of metrics of the form $g=\frac{d x^{2}}{\left(1+|x|^{2}+|\xi|^{2}\right)^{\ell}}+\frac{d \xi^{2}}{\left(1+|x|^{2}+|\xi|^{2}\right)^{\ell}}$ from which we can consider an operator of the form $K\left(x, D_{x}\right)=-\left(1+|x|^{2}-\Delta\right)^{\ell}$ which is of order $2 \ell$ but only of order 1 with respect to the metric (3.9).

We also note that the uncertainty principle is not satisfied if $\ell>1$, what makes the metric (3.9) not allowed in our setting in that case.

\subsection{Case of order $\nu=2$}

We shall now consider parabolic equations of order 2 . We start by proving a version of Theorem 3.2 when $K$ is of order 2 and scalar. We first establish a corresponding energy estimate. In the following theorems we will be considering operators $P\left(x, D_{x}\right) \in O p S\left(\lambda_{g}^{\nu}, g\right)$ satisfying the following condition with respect to a Hörmander metric $g$ and $\nu \in \mathbb{R}$ :

$(E, g, \nu)$ There exists a positive constant $C_{\nu}$ such that

$$
\operatorname{Re} P(x, \xi) \geq C_{\nu}\left(\lambda_{g}^{\nu}-1\right)
$$

We note that $\lambda_{g}^{\nu}-1 \geq 0$ due to the uncertainty principle. The condition $(E, g, \nu)$ is an extension of the notion of strongly ellipticity in the classes $S_{1,0}^{\nu}$. For the metric $g=g^{1,0}$ and $\nu=2$, we have $\lambda_{g}^{2}=\langle\xi\rangle^{2}$, and the ellipticity of order 2 for $K \in S_{1,0}^{2}$ is equivalent to the condition $\left(E, g^{1,0}, 2\right)$. Some other examples are given at the end of this section. 
Theorem 3.3. Let $g$ be a Hörmander metric, $s \in \mathbb{R}, T>0, K(t, x, \xi) \in$ $S\left(\lambda_{g}^{2}, g\right)$ depending smoothly on $t$ and $-K$ satisfying the condition $(E, g, 2)$. Let $Q=\frac{\partial}{\partial t}-K\left(t, x, D_{x}\right), v \in C^{1}\left([0, T], H\left(\lambda_{g}^{s}, g\right)\right) \bigcap C\left([0, T], H\left(\lambda_{g}^{s+1}, g\right)\right)$. Then, there exists a constant $C>0$ such that $v$ satisfies the energy estimate:

$$
\begin{aligned}
& \|v(t)\|_{H\left(\lambda_{g}^{s}, g\right)}^{2}+\int_{0}^{t}\|v(\tau)\|_{H\left(\lambda_{g}^{s+1}, g\right)}^{2} d \tau \\
& \quad \leq C\left(\|v(0)\|_{H\left(\lambda_{g}^{s}, g\right)}^{2}+\int_{0}^{t}\|Q v(\tau)\|_{H\left(\lambda_{g}^{s-1}, g\right)}^{2} d \tau\right)
\end{aligned}
$$

for all $t \in[0, T]$. The same conclusion holds for the operator $Q^{*}$.

Proof. First we will obtain some $L^{2}$ estimates. We write $Q v=\omega$, as in the proof of Theorem 3.1 one can prove that

$$
\begin{aligned}
\frac{d}{d t}\|v(t)\|_{L^{2}}^{2}= & 2 \operatorname{Re}\left\langle\partial_{t} v, v\right\rangle \\
= & 2 \operatorname{Re}\langle K(t) v, v\rangle \\
& +2 \operatorname{Re}\langle\omega, v\rangle .
\end{aligned}
$$

The term (3.12) can be estimated by applying the Fefferman-Phong inequality ([15], Theorem 18.6.8) to the nonnegative symbol $-K(t)+C_{2}-C_{2} \lambda_{g}^{2}$. We have

$$
\begin{aligned}
\operatorname{Re}\langle K(t) v, v\rangle & =\operatorname{Re}\left\langle\left(K(t)-C_{2}+C_{2} \Lambda_{g}^{2}+C_{2}-C_{2} \Lambda_{g}^{2}\right) v, v\right\rangle \\
& \leq C\|v\|_{L^{2}}^{2}+C_{3}\|v\|_{L^{2}}^{2}-C_{2}\|v\|_{H\left(\lambda_{g}, g\right)}^{2} \\
& \leq C\|v\|_{L^{2}}^{2}-C_{2}\|v\|_{H\left(\lambda_{g}, g\right)}^{2} .
\end{aligned}
$$

For (3.13), we have

$$
\begin{aligned}
|\langle v, \omega\rangle| & \leq\|v\|_{H\left(\lambda_{g}, g\right)}\|\omega\|_{H\left(\lambda_{g}^{-1}, g\right)} \\
& \leq \frac{1}{2}\left(\|v\|_{H\left(\lambda_{g}, g\right)}^{2}+\|\omega\|_{H\left(\lambda_{g}^{-1}, g\right)}^{2}\right)
\end{aligned}
$$

Then, by (3.14), (3.15) we obtain

$$
\frac{d}{d t}\|v(t)\|_{L^{2}}^{2} \leq 2 C\|v(t)\|_{L^{2}}^{2}-2 C_{2}\|v(t)\|_{H\left(\lambda_{g}, g\right)}^{2}+\|v(t)\|_{H\left(\lambda_{g}, g\right)}^{2}+\|\omega(t)\|_{H\left(\lambda_{g}^{-1}, g\right)}^{2} .
$$

We now temporarily assume $C_{2}>\frac{1}{2}$. Then $C_{2}-\frac{1}{2}=C_{0}>0$ and we have

$$
\frac{d}{d t}\|v(t)\|_{L^{2}}^{2}+C_{0}\|v(t)\|_{H\left(\lambda_{g}, g\right)}^{2} \leq C\|v(t)\|_{L^{2}}^{2}+\|\omega(t)\|_{H\left(\lambda_{g}^{-1}, g\right)}^{2} .
$$


Integrating between 0 and $t$ the above inequality we get

$$
\begin{aligned}
& \|v(t)\|_{L^{2}}^{2}+C_{0} \int_{0}^{t}\|v(\tau)\|_{H\left(\lambda_{g}, g\right)}^{2} d \tau \\
& \quad \leq C\left(\|v(0)\|_{L^{2}}^{2}+\int_{0}^{t}\left(\|v(\tau)\|_{L^{2}}^{2}+\|\omega(\tau)\|_{H\left(\lambda_{g}^{-1}, g\right)}^{2}\right) d \tau\right) .
\end{aligned}
$$

Therefore, replacing $v$ by $\Lambda_{g}^{s} v$ we have

$$
\begin{aligned}
& \|v(t)\|_{H\left(\lambda_{g}^{s}, g\right)}^{2}+C_{0} \int_{0}^{t}\|v(\tau)\|_{H\left(\lambda_{g}^{s+1}, g\right)}^{2} d \tau \\
& \quad \leq C\left(\|v(0)\|_{H\left(\lambda_{g}^{s}, g\right)}^{2}+\int_{0}^{t}\left(\|v(\tau)\|_{H\left(\lambda_{g}^{s}, g\right)}^{2}+\left\|Q \Lambda_{g}^{s} v(\tau)\right\|_{H\left(\lambda_{g}^{-1}, g\right)}^{2}\right) d \tau\right) .
\end{aligned}
$$

We now observe that $\Lambda_{g}^{-1} Q \Lambda_{g}^{s} v=\Lambda_{g}^{s-1} Q v+O\left(\|v\|_{H\left(\lambda_{g}^{s}, g\right)}\right)$. Thus, applying this identity to the second term in the integral of the right-hand side of (3.17) we get

$$
\begin{aligned}
& \|v(t)\|_{H\left(\lambda_{g}^{s}, g\right)}^{2}+C_{0} \int_{0}^{t}\|v(\tau)\|_{H\left(\lambda_{g}^{s+1}, g\right)}^{2} d \tau \\
& \quad \leq C\left(\|v(0)\|_{H\left(\lambda_{g}^{s}, g\right)}^{2}+\int_{0}^{t}\left(\|v(\tau)\|_{H\left(\lambda_{g}^{s}, g\right)}^{2}+\|\omega(\tau)\|_{H\left(\lambda_{g}^{s-1}, g\right)}^{2}\right) d \tau\right) .
\end{aligned}
$$

Now by Lemma 4.4, p. 363 of [5] we obtain

$$
\begin{aligned}
& \|v(t)\|_{H\left(\lambda_{g}^{s}, g\right)}^{2}+\int_{0}^{t}\|v(\tau)\|_{H\left(\lambda_{g}^{s+1}, g\right)}^{2} d \tau \\
& \quad \leq 2 C\left(\|v(0)\|_{H\left(\lambda_{g}^{s}, g\right)}^{2}+\int_{0}^{t}\|\omega(\tau)\|_{H\left(\lambda_{g}^{s-1}, g\right)}^{2} d \tau\right) .
\end{aligned}
$$

We have proved the estimate (3.11) assuming $C_{2}>\frac{1}{2}$. If $0<C_{2} \leq \frac{1}{2}$ then $\widetilde{K}=C_{2}^{-1} K$ satisfies the condition $(E, g, 2)$ with constant $C_{2}^{\prime}=1$ and consequently the estimate (3.11). for the operator $Q=\frac{\partial}{\partial t}-\widetilde{K}\left(t, x, D_{x}\right)$ holds. A time re-scaling argument shows that the energy estimate also holds for $Q=$ $\frac{\partial}{\partial t}-K\left(t, x, D_{x}\right)$ when $0<C_{2} \leq \frac{1}{2}$. This concludes the proof of the estimate (3.11). The fact that the estimate also holds for $Q^{*}$ follows analogously taking into account that $K^{*}$ also satisfies the condition $(E, g, 2)$.

Reasoning analogously as in the proof of Theorem 3.2 by using instead the above energy inequality we obtain the following theorem. 
Theorem 3.4. Let $g$ be a Hörmander metric, $s \in \mathbb{R}, T>0, K(t, x, \xi) \in$ $S\left(\lambda_{g}^{2}, g\right)$ depending smoothly on $t$ and $-K$ satisfying the condition $(E, g, 2)$. Let $f \in H\left(\lambda_{g}^{s}, g\right)$ and $\omega \in L^{2}\left([0, T], H\left(\lambda_{g}^{s-1}, g\right)\right)$. Then, there exists a unique solution $v \in C\left([0, T], H\left(\lambda_{g}^{s}, g\right)\right)$ of the Cauchy problem

$$
\left\{\begin{aligned}
\frac{\partial v}{\partial t} & =K(t) v+\omega, \quad\left(\text { in the sense of } \mathcal{D}^{\prime}(] 0, T\left[\times \mathbb{R}^{n}\right)\right) \\
v(0) & =f .
\end{aligned}\right.
$$

Moreover, the solution $v$ satisfies the energy estimate (3.11). If $\omega \in C^{\infty}$ $\left([0, T], H_{g}^{+\infty}\right)$ and $f \in H_{g}^{+\infty}$ then $v \in C^{\infty}\left([0, T], H_{g}^{+\infty}\right)$.

Example 2. One can also consider order 2 versions of operators $K$ as in Examples 1 and some others such that $-K$ satisfies the condition $(E, g, 2)$ with $C_{2} \geq 1$ :

(i) For the metric $g$ in Examples 1 (i), an example of an operator operator of order 2 is given by $L_{(g)}^{2}$ :

$$
L_{(g)}^{2}=\left(L+J^{\tau}\right)^{2} J^{-2}+R(x, D),
$$

where $R(x, D) \in O p S\left(\left(\frac{a(x, \xi)+\langle\xi\rangle^{\tau}}{\langle\xi\rangle}\right), g\right)$.

One can then consider $-K$ of the form $\left(L^{2}+J^{2 \tau}\right) J^{-2}$.

(ii) For $g$ as in Examples 1 (ii) we can consider for instance $K$ of order 2 defined by

$$
K\left(x, D_{x}\right)=-\left(-\Delta+|x|^{2}+I\right)^{2 \ell}
$$

or

$$
K\left(x, D_{x}\right)=-\left((-\Delta)^{2 \ell}+|x|^{2 \ell}\right),
$$

where $0<\ell \leq 1$.

Hence, one can also consider $K$ of the for $K=-\gamma(t)\left(-\Delta+|x|^{2}+\right.$ $I)^{2 \ell}$ with $0<c \leq \gamma \in C^{\infty}(\mathbb{R})$. We note that since $0<\ell \leq 1$, the order of the differential operator $K$ vary in the range $(0,4]$.

(iii) Let us now consider $K(x, D)=\partial_{x_{1}}^{2}+\widetilde{x}_{1}^{2} \partial_{x_{2}}^{2}-C$ on $\mathbb{R}^{2}$, with $C>0$ large enough, as presented in the introduction. It is known that $K(x, \xi) \in$ $S\left(\lambda_{g}^{2}, g\right)$ (cf. [29]) where $g$ is given by (3.7) and $\tau=1$. On the other hand we can see that $-K(x, \xi)$ verifies the condition $(E, g, 2)$. Indeed,

$$
\begin{aligned}
\lambda_{g}^{2}= & \frac{\left(\xi_{1}^{2}+\widetilde{x}_{1}^{2} \xi_{1}^{2}+\langle\xi\rangle\right)^{2}}{\langle\xi\rangle^{2}} \\
& \leq C \frac{\left(\xi_{1}^{4}+\widetilde{x}_{1}^{4} \xi_{2}^{4}+\xi_{1}^{2}+\xi_{2}^{2}+1\right)}{\xi_{1}^{2}+\xi_{2}^{2}+1} \\
& =C\left(\frac{\xi_{1}^{4}}{\xi_{1}^{2}+\xi_{2}^{2}+1}+\frac{\widetilde{x}_{1}^{4} \xi_{2}^{4}}{\xi_{1}^{2}+\xi_{2}^{2}+1}+1\right) \\
& \leq C\left(\xi_{1}^{2}+\widetilde{x}_{1}^{4} \xi_{2}^{2}+1\right) \\
& \leq C\left(\xi_{1}^{2}+\widetilde{x}_{1}^{2} \xi_{2}^{2}+1\right) .
\end{aligned}
$$

Therefore

$$
\lambda_{g}^{2}-1 \leq C\left(\xi_{1}^{2}+\widetilde{x}_{1}^{2} \xi_{2}^{2}+C^{\prime}\right)
$$


which proves the ellipticity condition $(E, g, 2)$.

The case of the fractional Laplacian requires further analysis and it will be treated in the following section.

\subsection{The fractional Laplacian}

We shall now consider the case of fractional diffusion as has been announced in the introduction. This example will require of some preliminary adaptation since the fractional Laplacian can not be directly treated as a pseudodifferential operator. Indeed, for $0<s<1$ the fractional Laplacian $(-\Delta)^{s} 0<$ $s<1$ has not a symbol in a class of symbols for pseudo-differential operators. We recall that $(-\Delta)^{s}$ is defined by

$$
\widehat{(-\Delta)^{s}} u(\xi)=|\xi|^{2 s} \widehat{u}(\xi), u \in \mathcal{S}\left(\mathbb{R}^{n}\right) .
$$

There are several equivalent definitions of the fractional Laplacian. We refer the reader to [17] for a recent review on the most common ones and the equivalence between them.

For more details on the following decomposition we refer to [12]. In order to apply the pseudo-differential calculus to the fractional Laplacian we choose a cut-off function $\varphi: \mathbb{R}^{n} \rightarrow[0,1]$ such that

$$
\varphi(\xi)=0 \text { for }|\xi|<1 \text { and } \varphi(\xi)=1 \text { for }|\xi|>2 .
$$

We consider the classical symbol defined by

$$
\widetilde{a}(\xi):=|\xi|^{2 s} \varphi(2 \xi), \text { for all } \xi \in \mathbb{R}^{n} .
$$

One can prove that $\widetilde{a} \in S_{1,0}^{m}$ for $m=1 \vee 2 s$ and

$$
(-\Delta)^{s} u=\widetilde{a}(D) u+E u, u \in \mathcal{S}\left(\mathbb{R}^{n}\right),
$$

where $E$ is defined by

$$
E u(x):=\mathcal{F}^{-1}\left[|\xi|^{2 s}(1-[\varphi(\xi)) \widehat{u}(\xi)], u \in \mathcal{S}\left(\mathbb{R}^{n}\right) .\right.
$$

Hereafter we will assume $\frac{1}{2}<s<1$. In order to extend the operator $(-\Delta)^{s}$ to a larger subspace of $\mathcal{S}^{\prime}\left(\mathbb{R}^{n}\right)$ one considers

$$
\mathcal{S}_{(0, s)}^{\prime}\left(\mathbb{R}^{n}\right):=\left\{u \in \mathcal{S}^{\prime}\left(\mathbb{R}^{n}\right):|\xi|^{2 s} \widehat{u} \in \mathcal{S}^{\prime}\left(\mathbb{R}^{n}\right)\right\} .
$$

From (3.22) and since $\widetilde{a}(D)$ is a classical pseudo-differential operator one obtains

$$
(-\Delta)^{s}: \mathcal{S}_{(0, s)}^{\prime}\left(\mathbb{R}^{n}\right) \rightarrow \mathcal{S}^{\prime}\left(\mathbb{R}^{n}\right)
$$

We will also need

$$
\widehat{L}_{l o c}^{2}\left(\mathbb{R}^{n}\right):=\left\{u \in \mathcal{S}^{\prime}\left(\mathbb{R}^{n}\right): \widehat{u} \in L_{l o c}^{2}\left(\mathbb{R}^{n}\right)\right\} .
$$

We recall that a tempered distribution, $u \in \mathcal{S}^{\prime}\left(\mathbb{R}^{n}\right)$ belongs to the Sobolev space $H^{\gamma}\left(\mathbb{R}^{n}\right)$, where $\gamma \in \mathbb{R}$, if $\langle\xi\rangle^{\gamma} \widehat{u}(\xi) \in L^{2}\left(\mathbb{R}^{n}\right)$. We also recall that for the metric $g=g^{\rho, \delta}$ one has $H\left(\lambda_{g}^{\gamma}, g\right)=H^{\gamma}\left(\mathbb{R}^{n}\right)$, for all $\gamma \in \mathbb{R}$. We say that $u \in H^{\gamma}\left(\mathbb{R}^{n}\right)$, if for any function $\chi \in \mathcal{C}_{c}^{\infty}\left(\mathbb{R}^{n}\right)$, we have that $\chi u$ belongs to $H^{\gamma}\left(\mathbb{R}^{n}\right)$. Using the facts that $1-\varphi(2 \xi)$ is a compactly supported, smooth function, and

$$
\langle\xi\rangle^{\gamma} \widehat{E u}(\xi)=\langle\xi\rangle^{\gamma}|\xi|^{2 s}(1-\varphi(2 \xi)) \widehat{u}(\xi), \forall m \in \mathbb{R},
$$


we obtain that if $u \in \widehat{L}_{l o c}^{2}\left(\mathbb{R}^{n}\right)$, then $\langle\xi\rangle^{\gamma} \widehat{E u}(\xi) \in L^{2}\left(\mathbb{R}^{n}\right)$.

Therefore

$$
E: \widehat{L}_{l o c}^{2}\left(\mathbb{R}^{n}\right) \rightarrow H^{\gamma}\left(\mathbb{R}^{n}\right), \text { for all } \gamma \in \mathbb{R}
$$

On the other hand we recall that the Hörmander metric $g^{\rho, \delta}$ is defined by

$$
g^{\rho, \delta}(d x, d \xi)=\langle\xi\rangle^{2 \delta} d x^{2}+\frac{d \xi^{2}}{\langle\xi\rangle^{2 \rho}},
$$

where $0 \leq \delta \leq \rho \leq 1$ and $\delta<1$. For the metrics $g=g^{\rho, \delta}$ we have $\lambda_{g}(x, \xi)=$ $\langle\xi\rangle^{\rho-\delta}$. If $\delta<\rho$ an example of an operator of order $\mu \in \mathbb{R}$ is given by $L_{(g)}^{\mu}$ of the form

$$
L_{(g)}^{\mu}=\Lambda^{\mu(\rho-\delta)}+R(x, D)
$$

where $R(x, D) \in O p S\left(\langle\xi\rangle^{(\mu-1)(\rho-\delta)}, g^{\rho, \delta}\right)$. In particular, $L_{(g)}^{2}=\Lambda^{2(\rho-\delta)}$ is of order 2 .

We now write $s=\rho-\delta$ with $\frac{1}{2}<\rho-\delta<1$. We note that $\widetilde{a}$ satisfies the ellipticity condition $(E, g, 2)$. Indeed,

$$
\widetilde{a}+1 \approx\langle\xi\rangle^{2 s}=\langle\xi\rangle^{2(\rho-\delta)} .
$$

Hence,

$$
\widetilde{a} \geq C\left(\langle\xi\rangle^{2(\rho-\delta)}-1\right)
$$

We will now take care of the operator $E$. A close look to the proof of the energy estimate given by Theorem 3.1 shows to us that we can extend the energy inequality to $\widetilde{a}(D)+E$ as a consequence of the boundedness (3.24). Indeed, one has the following mild lemma.

Lemma 3.5. Let $E$ be the operator as in (3.23). Then

$$
\|E v\|_{H^{\gamma}} \leq C\|v\|_{H^{\gamma}}, \text { for all } \gamma \in \mathbb{R}
$$

Proof. We first note that from (3.24) for $\gamma=0$ we have

$$
\|E v\|_{L^{2}} \leq C\|v\|_{L^{2}}, \text { for all } .
$$

On the other hand, since $E$ and $\Lambda^{\gamma}$ commute and by (3.24) we have

$$
\|E v\|_{H^{\gamma}}^{2}=\left\|\Lambda^{\gamma} E v\right\|_{L^{2}}^{2}=\left\|E \Lambda^{\gamma} v\right\|_{L^{2}}^{2} \leq C\left\|\Lambda^{\gamma} v\right\|_{L^{2}}^{2}=\|v\|_{H^{\gamma}}^{2} .
$$

The lemma above allows to handle the additional term $E$ without losing the previous energy estimate. Now, if $\frac{1}{2}<s<1$ we can consider the metric (3.25). By writing $s=\frac{\rho-\delta}{2}$ we cover $s$ in the interval $\left(\frac{1}{2}, 1\right)$ and we can extend Theorem 3.3: 
Theorem 3.6. Let $\frac{1}{2}<s<1, T>0, \gamma \in \mathbb{R}, v \in C^{1}\left([0, T], H^{\gamma}\right) \bigcap C([0, T]$, $\left.H^{\gamma+1}\right)$ and $Q=\frac{\partial}{\partial t}+(-\Delta)^{s}$. Then, there exists a constant $C>0$ such that $v$ satisfies the energy estimate:

$$
\begin{aligned}
& \|v(t)\|_{H^{\gamma}}^{2}+\int_{0}^{t}\|v(\tau)\|_{H^{\gamma+1}}^{2} d \tau \\
& \leq C\left(\|v(0)\|_{H^{\gamma}}^{2}+\int_{0}^{t}\|Q v(\tau)\|_{H^{\gamma-1}}^{2} d \tau\right)
\end{aligned}
$$

for all $t \in[0, T]$. The same conclusion holds for the operator $Q^{*}$.

Consequently, we can state the following extension of Theorem 3.4 for the fractional diffusion.

Theorem 3.7. Let $T>0, \frac{1}{2}<s<1, \gamma \in \mathbb{R}, f \in H^{\gamma}$ and $\omega \in L^{2}\left([0, T], H^{\gamma-1}\right)$. Then, there exists a unique solution $v \in C\left([0, T], H^{\gamma}\right)$ of the Cauchy problem

$$
\left\{\begin{aligned}
\frac{\partial v}{\partial t} & =-(-\Delta)^{s} v+\omega, \quad\left(\text { in the sense of } \mathcal{D}^{\prime}(] 0, T\left[\times \mathbb{R}^{n}\right)\right), \\
v(0) & =f,
\end{aligned}\right.
$$

Moreover, the solution $v$ satisfies the energy estimate (3.26). If $\omega \in$ $C^{\infty}\left([0, T], H^{+\infty}\right)$ and $f \in H^{+\infty}$ then $v \in C^{\infty}\left([0, T], H^{+\infty}\right)$.

\section{Acknowledgements}

The author wants to thank the anonymous referee for the interesting comments. The author would also like to thank Prof. Michael Ruzhansky for many inspiring discussions.

Open Access. This article is distributed under the terms of the Creative Commons Attribution 4.0 International License (http://creativecommons.org/licenses/ by/4.0/), which permits unrestricted use, distribution, and reproduction in any medium, provided you give appropriate credit to the original author(s) and the source, provide a link to the Creative Commons license, and indicate if changes were made.

\section{References}

[1] Barbatis, G., Gazzola, F.: Higher order linear parabolic equations. In: Recent trends in nonlinear partial differential equations. I. Evolution Problems. Contemporary Mathematics, vol. 594, pp. 77-97. American Mathematical Society, Providence, RI (2013)

[2] Beals, R., Gaveau, B., Greiner, P.: Green's functions for some highly degenerate elliptic operators. J. Funct. Anal. 165, 407-429 (1999)

[3] Bony, J.M., Chemin, J.Y.: Espaces fonctionnels associés au calcul de WeylHörmander. Bull. Soc. Math. Fr. 122, 77-118 (1994)

[4] Bony, J.-M., Lerner, B.: Quantification asymptotique et microlocalisation d'ordre superieur I. Ann. Sci. Ec. Norm. Sup. 22, 377-433 (1989) 
[5] Chazarain, J., Piriou, A.: Introduction to the Theory of Linear Partial Differential Equations. North-Holland, Oxford (1982)

[6] Constantin, P.: Navier-Stokes equations and turbulence. In: Constantin, P., et al. (eds.) Mathematical Foundation of Turbulent Viscous Flows. Lecture Notes in Mathematics, 1, vol. 43, p. 1871. Springer, Berlin (2006)

[7] Cont, R., Tankov, P.: Financial Modelling with Jump Processes. Financial Mathematics Series. Chapmann and Hall/CRC, Boca Raton (2004)

[8] Delgado, J.: A class of invertible subelliptic operators in $S(m, g)$-classes. Results Math. 67(33), 431-444 (2015)

[9] Delgado, J.: On a class of hyperbolic equations in Weyl-Hörmander calculus. J. Math. Anal. Appl. 436(1), 339-354 (2016)

[10] Delgado, J., Zamudio, A.: Invertibility for a class of degenerate elliptic operators. J. Pseudo Differ. Oper. Appl. 1(2), 207-231 (2010)

[11] DiBenedetto, E.: Degenerate parabolic equations. Springer, New York (1993)

[12] Epstein, C., Pop, C.: Regularity for the supercritical fractional Laplacian with drift. J. Geom. Anal. 26(2), 1231-1268 (2016)

[13] Gazzola, F., Grunau, H.C.: Some new properties of biharmonic heat kernels. Nonlinear Anal. 70, 2965-2973 (2009)

[14] Hörmander, L.: The Weyl calculus of pseudodifferential operators. Commun. Pure Appl. Math. 32, 359-443 (1979)

[15] Hörmander, L.: The Analysis of Linear Partial Differential Operators, vol. III. Springer, Berlin (1985)

[16] Kohn, J., Nirenberg, L.: An algebra of pseudo-differential oprators. Commun. Pure Appl. Math. 18(1-2), 269-305 (1965)

[17] Kwasnicki, M.: Ten equivalent definitions of the fractional laplace operator. Fract. Calc. Appl. Anal. 20(1), 7-51 (2017)

[18] Laskin, N.: Fractional quantum mechanics. Phys. Rev. E 62, 3135-3145 (2000)

[19] Laskin, N.: Principles of Fractional Quantum Mechanics. Fractional Dynamics, pp. 393-427. World Sci. Publ, singapore (2012)

[20] Lerner, N.: Metrics on the Phase Space and Non-Selfadjoint Pseudo-Differential Operators. Pseudo-Differential Operators. Birkhäuser, Basel (2010)

[21] Lindsay, A.: An asymptotic study of blow up multiplicity in fourth order parabolic partial differential equations. Discrete Contin. Dyn. Syst. Ser. B. 19(1), 189-215 (2014)

[22] Majda, A.J., Bertozzi, A.L.: Vorticity and Incompressible Flow. Cambridge Texts in Applied Mathematics. Cambridge Univ. Press, Cambridge (2002)

[23] Nicola, F.: Phase space analysis of semilinear parabolic equations. J. Funct. Anal. 267(3), 727-743 (2014)

[24] Parenti, C., Parmeggiani, A.: Lower bounds for systems with double characteristics. J. Anal. Math. 86, 49-91 (2002)

[25] Parmeggiani, A.: Subunit balls for symbols of pseudodifferential operators. Adv. Math. 131(2), 357-452 (1997)

[26] Sokolov, J.K.I.M., Blumen, A.: Fractional kinetics. Phys. Today 55, 48-54 (2002)

[27] Taylor, M.E.: Pseudodifferential Operators. Princeton Mathematical Series, vol. 34. Princeton University Press, Princeton (1981) 
[28] Taylor, M.E.: Partial Differential Equations II: Qualitative Stuties of Linear Equations. Springer, New York (2011)

[29] Xu, C., Zhu, X.: On the inverse of a class of degenerate elliptic operator. Chin. J. Contemp. Math. 16(3), 261-274 (1995)

\author{
Julio Delgado $(\bowtie)$ \\ Department of Mathematics \\ Imperial College London \\ 180 Queen's Gate \\ London SW7 2AZ \\ UK \\ e-mail: j.delgado@imperial.ac.uk
}

Received: June 23, 2017.

Revised: January 11, 2018. 